\title{
CONSECUENCIAS ALTERNATIVAS: LA IMPORTANCIA DE SU CONOCIMIENTO EN LA IMPLEMENTACIÓN DE POLÍTICAS
}

\author{
Leonardo Ivarola
}

\begin{abstract}
Resumen: En el presente trabajo se examinarán las distintas clases de conocimientos que se requieren para una correcta implementación de política. Cartwright y Hardie (2012) han criticado el enfoque de la política basada en la evidencia por estar fundamentado en inferencias inductivas, y han propuesto un enfoque alternativo donde, por un lado, el principio causal o variable política se abstraiga lo máximo posible como para superar cualquier problema de validez externa, y por el otro, que este principio sea complementado con un acervo de factores coadyuvantes (los cuales refieren a aspectos muy concretos de un sistema objetivo). Si bien este enfoque proporciona una buena base para el armado de políticas, omite un tema central: las consecuencias alternativas que se derivan de una política. Más precisamente, en el enfoque de Cartwright y Hardie se apunta a recolectar información a fin de armar una política que funcione aquí, pero no dice nada si la política se desvía de su curso deseado. El problema de esto emerge cuando los desvíos conllevan consecuencias trascendentalmente negativas. Se argumentará que el conocimiento de las consecuencias alternativas de una política es tan importante como el conocimiento de factores centrales y de factores coadyuvantes. La toma de decisión de un hacedor de política no dependerá solo entonces de las chances de éxito de una política, sino también de la asimetría de sus resultados (TALEB, 2012).
\end{abstract}

Palabras Clave: Implementación de políticas. Asimetría. Resultados alternativos. Política basada en la evidencia.

\section{INTRODUCCIÓN}

La politica basada en la evidencia (PBE) es un enfoque cuyo principio fundamental es el de utilizar políticas que funcionaron en otro lugar.

\footnotetext{
${ }^{1}$ Profesor Adjunto de la materia Epistemología de la Economía en la Universidad de Buenos Aires, Buenos Aires - Argentina. (D) https://orcid.org/0000-0002-9997-3720 E-mail: ivarola@economicas.uba.ar

Licenciado en Economía y Doctor en Filosofía por la Universidad de Buenos Aires. Ha publicado artículos en revistas tanto nacionales como internacionales. Ha sido becario doctoral UBACyT y becario posdoctoral de CONICET. Actualmente es investigador asistente de CONICET con lugar de trabajo en el Centro de Investigaciones en Epistemología de las Ciencias Económicas (CIECE). Es miembro del Comité Ejecutivo de las Jornadas de Epistemología de las Ciencias Económicas.
}

http://dx.doi.org/10.1590/0101-3173.2019.v42n2.10.p195

This is an open-access article distributed under the terms of the Creative Commons Attribution License. 
Dicho enfoque ha estado siendo utilizado de modo creciente en los últimos años, sobre todo en el campo de las políticas sociales. Cartwright (2012) y Cartwright y Hardie (2012) han criticado dicha metodología, sugiriendo que las afirmaciones causales a las que apela $\mathrm{PBE}$ refieren a cosas que han funcionado en alguna parte. Sin embargo, para que una política sea exitosa, no es ni necesario ni suficiente saber esto. Lo relevante, en cambio, estriba en conocer si una política funcionará aqui y ahora.

Para que esto pueda darse, Cartwright y Hardie (2012) sugieren dos modos de investigación: la investigación vertical y la horizontal. La investigación vertical consiste en describir el principio causal central (o variable política) en el grado de abstracción correcto. La investigación horizontal consiste en explicitar el acervo de factores coadyuvantes que complementan ese principio causal. Mientras que el principio causal es extrapolable a cualquier escenario, los factores coadyuvantes refieren a niveles mayores de concretización, esto es, son específicos de cada política. El conjunto del principio causal y de factores coadyuvantes nos permiten armar una "torta causal" cuyos componentes actúan como condiciones INUS: cada elemento de la torta es condición necesaria (pero no suficiente) para alcanzar un resultado, al tiempo que cada torta es suficiente (pero no necesaria) para alcanzarlo.

Si bien el enfoque de Cartwright y Hardie (2012) arroja luz respecto de los condicionamientos para que una política sea efectiva aquí y ahora, no nos dice nada respecto de lo que podría pasar si las cosas fueran diferentes, esto es, si el proceso causal real se desviase del proceso descripto en la torta causal. Éste no es un tema menor. En algunas situaciones, las consecuencias alternativas de una "buena" decisión pueden ser sustancialmente perjudiciales.

En este sentido, el presente trabajo apunta a examinar, por un lado, las razones que pueden originar tales desvíos, y por el otro lado, qué se puede hacer con dicho conocimiento. Las políticas que se abordarán aquí serán principalmente económicas, aunque es plausible su extensión a otros campos de las ciencias sociales.

Un primer punto consiste en argumentar que los procesos causales que emergen de esta clase de políticas no son congruentes con una lógica de factores causales estables, sino con una lógica de resultados de final abierto. Se mostrará que la volatilidad de la toma de decisiones de las personas es crucial aquí, ya que cualquier cambio en la interpretación que éstas hacen respecto 
de las señales del mundo o en su formación de expectativas redundará en una modificación del proceso causal inicial.

Asimismo, se argumentará que el conocimiento de las consecuencias alternativas que se derivan del ejercicio de una política o "torta causal" es tan importante como el conocimiento que ésta proporciona respecto de su curso planeado. En primer lugar, un hacedor de política debe ser consciente de que a veces las cosas pueden no salir de acuerdo con lo esperado. En otras palabras, el sendero causal de una política puede desviarse hacia un camino inesperado. El problema estriba en si ese desvío conduce a consecuencias significativamente negativas. Una vez que el hacedor de política haga uso de este conocimiento, su toma de decisiones deberá tener en cuenta la asimetría de los resultados (TALEB, 2012). Si en la implementación de una política lo que se gana (si tiene éxito) es poco y lo que se pierde (si fracasa) es mucho, lo ideal sería no implementar dicha política, independientemente de sus chances de éxito. Lo inverso sucede si lo que se gana es mucho en comparación con lo que se puede llegar a perder.

El conocimiento que proporcionan las tortas causales refiere a aquello que sucedería en situaciones muy precisas. Sin embargo, es incapaz de decirnos qué pasaría si algún elemento no planeado tuviese lugar. Por tal razón, en el presente trabajo se apelará a un modelo económico cuya contribución causal es ampliamente utilizada en la implementación de políticas: el "efecto Keynes". En éste se hace una defensa de las políticas monetarias expansivas en escenarios que involucran recesión sin inflación. Sin embargo, se mostrará que el conocimiento que este modelo proporciona no sólo refiere a un escenario muy específico, sino que también pueden originarse múltiples desvíos si las personas cambian su toma de decisiones o si emerge alguna modificación en las condiciones del contexto. Es menester tener en consideración que, mientras que el hacedor de políticas puede controlar algunos de estos cambios, no puede tener un control supremo sobre todos ellos.

El trabajo se articula de la siguiente manera. En la siguiente sección se expondrán unas líneas generales del enfoque PBE, así como también la propuesta alternativa de Cartwright (2012) y Cartwright y Hardie (2012). En la tercera sección se argumentará que, dada la volatilidad de la acción humana y de ciertos factores del contexto, las políticas sociales en general y económicas en particular pueden desviarse de su curso deseado. Se propondrá como ejemplo un modelo muy utilizado en política económica: el "efecto Keynes”. En la cuarta y última sección se mostrará la importancia de conocer los desvíos o consecuencias alternativas de una política, en tanto éstas pueden 
desproporcionadas respecto de la política pensada. Se tomará como marco teórico de referencia la noción de "asimetría" propuesta por Taleb (2012).

\section{Más ALlá de LA POLÍTICA BASADA EN LA EVIDENCIA}

La "política basada en la evidencia" (PBE) es un enfoque que consiste en la utilización del conocimiento causal basado en el éxito de políticas pasadas. El principio de la $\mathrm{PBE}$ es el de usar políticas que funcionaron en algún lugar. En este marco, las pruebas de control aleatorio constituyen la herramienta clave de este enfoque. No obstante, Cartwright (2012) y Cartwright y Hardie (2012) argumentan que las pruebas de control aleatorio sólo proporcionan información respecto de un factor causal que ha funcionado en la población donde se hizo la prueba. Ahora bien, el interés que subyace a la implementación de una política está asociado con la relevancia del conocimiento causal para esta nueva política, y no para otras o para casos generales. En este sentido, Cartwright (2012) y Cartwright y Hardie (2012) diferencian entre tres tipos de afirmaciones causales:

1. funciona en alguna parte

2. funciona en general

3. funcionará aquí y ahora,

las cuales los autores consideran que son ineludibles si se pretende tener éxito en la implementación de una política. Es necesario conocer cómo bajo determinadas circunstancias un factor causal produce un efecto (funciona en alguna parte); esta es la piedra angular de la PBE. Sin embargo, de ello no se sigue que funcionará para el caso objetivo (funcionará aqui y ahora), y mucho menos que funcionará siempre (funciona en general).

Tomemos como ejemplo dos políticas para combatir la desnutrición infantil. Hacia fines del siglo XX, en el sur de Asia el Banco Mundial estuvo implementado programas para combatir la desnutrición y malnutrición infantil. Uno de estos programas fue el Proyecto de nutrición integrado de Bangladesh (Bangladesh integrated nutrition project o BINP), el cual fue iniciado en respuesta no sólo a un muy alto nivel de malnutrición prevaleciente en el país, sino también al hecho de que las actividades focalizadas explícitamente en nutrición habían sido olvidadas tanto por el gobierno como por las agencias externas (BANCO MUNDIAL, 2005). 
El BINP fue modelado sobre la base de un programa llevado a cabo en la India: el Indian Tamil Nadu integrated nutrition proyect (TINP), con poco esfuerzo para adaptar el proyecto a circunstancias locales (BANCO MUNDIAL, 2005). El TINP cubría las áreas rurales de los distritos con los peores índices de desnutrición, el cual involucraba a prácticamente la mitad del estado de Tamil Nadu. El programa fue efectivo, haciendo caer considerablemente los índices de desnutrición.

No obstante, el BINP no logró el éxito del TINP. Uno de los principales objetivos del BINP era mejorar el estatus nutricional de mujeres embarazadas y en periodos de lactancia, así como también de infantes en las comunidades más pobres de Bangladesh. Para el logro de ello, el BINP proporcionó asesoramiento nutricional a mujeres embarazadas y alimentación suplementaria para niños debajo de los 24 meses. Se esperaba que dicho asesoramiento fuera efectivo no sólo para mejorar los índices de malnutrición, sino también para provocar en el mediano plazo una transformación de ciertas normas y creencias de los habitantes de Bangladesh. ${ }^{2}$

¿Por qué no funcionó el BINP, si el TINP logró tener éxito? Hay dos factores que contribuyeron a esto: la filtración y la sustitución de alimentos. En primer lugar, la comida pensada como suplementaria era en realidad usada como sustituta. Además, ciertos alimentos destinados a las madres y nińos terminaba siendo "filtrada" hacia otros miembros de la familia. Estos dos factores están vinculados con un patrón sociocultural muy significativo para el resultado de la política: el control de las madres en el hogar. El TINP era un programa pensado para la cultura de la India, donde las mujeres son las encargadas de la administración del hogar. Pero en Bangladesh esto no es así. Por el contrario, son las suegras las que tienen el control. Las madres tampoco hacen las compras; son los padres quienes se encargan de ello. En la India, el factor "las madres tienen el control del hogar" no era significativo o saliente debido a su omnipresencia. Resultó ser significativo en Bangladesh, donde su ausencia contribuyó a la generación de resultados muy diferentes a los esperados.

Ahora bien, en lugar de preguntarnos “¿por qué falló el programa BINP?”, uno debería preguntarse "¿por qué deberíamos esperar a que tenga éxito?”. Una posible respuesta es "porque ha funcionado en alguna otra parte”. Éste es el supuesto fundamental de la política basada en la evidencia. No obstante, la mera extrapolación de políticas no está garantizada simplemente

${ }^{2}$ Por ejemplo, dentro de esta cultura existe la concepción de que, al comer menos, los bebés serán más pequeños, lo que hará más fácil la carga. 
porque está basada en una inferencia inductiva. En otras palabras, que una política haya tenido éxito en un escenario no es condición suficiente para que tenga éxito en otro diferente.

En contraposición al enfoque de PBE, Cartwright y Hardie (2012) afirman que, antes de implementar un programa, un hacedor de política debería llevar a cabo dos tipos de investigaciones: la investigación vertical y la investigación horizontal.

La investigación vertical consiste en averiguar si el factor causal central ha sido descripto en el nivel de abstracción correcto. Por ejemplo, el TINP resultó ser un programa exitoso, y sirvió de base para el diseño del BINP. No obstante, el resultado no fue el esperado. Cartwright y Hardie (2012) atribuyen ese fracaso no sólo a la omisión de factores contextuales, sino también a la incorrecta abstracción de principios causales subyacentes. Para comprender mejor este punto, consideremos el siguiente enunciado:

Principio 1: mejor conocimiento nutricional de las madres más suministro de alimentación suplementaria pueden mejorar el estatus nutricional de los niños.

Se trata de un principio basado en la observación de un programa exitoso en algún lugar ( $v . g r$., Tamil Nadu), pero no en otro ( $v . g r$., Bangladesh). Extrapolar este principio es un error, ya que hay presentes factores de un nivel de concreción que es aplicable a un sitio, pero no a otro. Por consiguiente, Cartwright y Hardie (2012) sugieren "ascender" hacia un principio más abstracto, como por ejemplo:

Principio 2: mayor conocimiento nutricional puede mejorar la nutrición de los niños si las personas que tienen el conocimiento son aquellas que

a. proveen al niño con alimentación suplementaria

b. controlan qué comida es adquirida

c. controlan cómo la comida es administrada

$\boldsymbol{d}$. mantienen los intereses del niño como central al llevar a cabo a, $\boldsymbol{b}$, y $\boldsymbol{c}$.

Este último principio es viable tanto para el caso de Tamil Nadu como para el de Bangladesh, ya que las especificaciones no están restringidas a escenarios específicos. Por ejemplo, este principio menciona que el programa debe brindar asesoramiento nutricional a aquél miembro de la familia que controle el hogar, sin especificar si es madre, padre o suegra. 
Ahora bien, las causas no operan en aislamiento, sino en conjunción con otros factores, a los que Cartwright y Hardie (2012) denominan "factores coadyuvantes". Esta es la tarea de la investigación horizontal. La investigación horizontal consiste en bajar en niveles de abstracción, a los efectos de especificar los elementos puntuales que serán cruciales para el éxito de una política en un contexto determinado. Aquí es cuando la investigación vertical se complementa con la horizontal. Saber que el aconsejar a la persona que distribuye la comida ayudará a la nutrición infantil no nos dice a quiénes se deben aconsejar en Tamil Nadu, en Bangladesh o en Dock Sud. Cada escenario hace uso de factores coadyuvantes diferentes. Si bien el principio causal puede ser el mismo para un conjunto de políticas, es menester investigar las propiedades específicas de cada sistema objetivo. ${ }^{3}$

Otra manera de entender la implementación de políticas es a través de la noción de "torta causal" (CARTWRIGHT; HARDIE, 2012). La variable política o factor causal central es un ingrediente de la torta, y juega su papel causal trabajando con otros ingredientes a fin de producir una contribución al efecto final. Estos ingredientes son lo que Cartwright y Hardie llaman "factores coadyuvantes". Dichos factores pueden entenderse como condiciones INUS: una parte insuficiente pero necesaria de una condición que es innecesaria pero suficiente para su efecto (Mackie 1965). Una condición INUS es un ingrediente o una parte de la torta. Sin esto, su contribución causal no tendría lugar. Por lo tanto, es una condición necesaria para producirla. Sin embargo, es una parte insuficiente, esto es, no es suficiente para producir una contribución causal por su cuenta, sino que necesita de otros factores o ingredientes que la apoyen. Por otro lado, cada torta se considera suficiente para producir una contribución causal. Sin embargo, esta contribución siempre se puede generar a partir de una variedad de tortas diferentes con diferentes ingredientes. Esa es la razón por la cual cada torta particular es también una condición innecesaria para conseguir una contribución al efecto. Cabe destacar también que las tortas causales son condición suficiente la el éxito de una política: si tanto la investigación horizontal como la vertical se llevan a cabo del modo correcto, el hacedor de políticas tendrá a su disposición todos aquellos factores que garanticen alcanzar el resultado deseado.

\footnotetext{
${ }^{3}$ Una variante de la investigación horizontal la proporciona Cartwright en su libro How the laws of physics lie (1983). Allí la autora muestra cómo varias causas se combinan para producir un determinado fenómeno.
} 


\section{La IMPLEMENTACIÓN DE UNA POLÍTICA Y SUS POSIBLES DESVÍOS: EL CASO DEL "EFECTO KeYNES"}

Supongamos que se desea implementar una política para aumentar el Producto Interno Bruto o Ingreso Nacional de un país. Los hacedores de política tienen a su disposición diferentes instrumentos macroeconómicos para lograr este objetivo. Algunos de estos instrumentos -como aumentar el gasto público o reducir los impuestos- están a cargo del poder ejecutivo y/o legislativo. Estos instrumentos pertenecen a lo que en economía se denomina "política fiscal". Alternativamente, los bancos centrales de un país son los encargados de llevar a cabo la "política monetaria", la cual consiste básicamente en alterar el nivel de base monetaria.

En economía existe un mecanismo que vincula los incrementos entre oferta monetaria e ingreso nacional, y se lo conoce como "efecto Keynes" (EK). De acuerdo con este mecanismo, un aumento en la cantidad de dinero $(M)$ puede conducir a un descenso en la tasa de interés ( $i)$, estimulando inversión $(I)$ y en consecuencia el empleo $(N)$ y la producción $(Y)$. Ahora bien, el EK no es un mecanismo que se activa siempre que aumente la oferta monetaria. Para que éste tenga lugar, ciertas condiciones deberán cumplirse, a saber:

1. existen rendimientos constantes a escala, de modo tal que los precios no aumentan (o caen) en la medida en que aumenta la producción

2. existe una relación causal negativa entre tasa de interés e inversión

3. la preferencia por la liquidez permanece constante, o al menos su incremento es menor al incremento en la cantidad de dinero

4. la demanda de dinero depende negativamente de la tasa de interés y positivamente del ingreso

5. la oferta de dinero es inelástica con respecto a la tasa de interés

6. cambios en la ocupación primaria en la industria de inversión provocan cambios proporcionalmente mayores en la ocupación total

7. la propensión marginal a consumir permanece constante ante cambios en el ingreso.

La primera etapa del EK (de $M$ hacia $i$ ) es generada por un aumento en la oferta monetaria. La explicación tradicional consiste en asumir que las 
personas aumentarán su demanda de bonos, el precio de éste aumentará, y como consecuencia la tasa de interés tenderá a caer. La segunda etapa (de $i$ hacia I) concierne a la demanda de inversión de las empresas. Si la tasa de interés cae, los créditos serán más baratos para el empresario. Como consecuencia de ello, la inversión aumentará. En lo que respecta a la última etapa (de $I$ hacia $N$ e $Y$ ), Keynes distingue entre ocupación primaria en las industrias de inversión $\left(N_{2}\right)$, y ocupación total $(N)$. Cambios en la inversión ocasionan cambios en $N_{2}$. Por otro lado, el multiplicador de Kahn (también denominado multiplicador de la ocupación) muestra cuánto aumentará la ocupación total ante un aumento en $N_{2}$. Supongamos entonces que un aumento en la inversión tiene lugar. Este aumento irá acompañado de un aumento en la ocupación primaria en las industrias de inversión. Ceteris paribus, y a través del multiplicador de la ocupación, esto debería aumentar la ocupación total.

Puesto que el EK es un mecanismo que depende del cumplimiento de ciertos factores, su funcionamiento puede ser detenido en tanto ciertos factores perturbadores tengan lugar. A este respecto, Keynes (1936, p.150) comenta:

$\mathrm{Si}$ bien puede esperarse que, ceteris paribus, un aumento en la cantidad de dinero reduzca la tasa de interés, esto no sucederá si las preferencias por la liquidez del público aumentan más que la cantidad de dinero; y mientras que puede esperarse que, ceteris paribus, un descenso en la tasa de interés aumente el volumen de la inversión, esto no ocurrirá si la curva de la eficiencia marginal del capital baja con mayor rapidez que la tasa de interés; y mientras es de suponer que, ceteris paribus, un aumento en el volumen de la inversión haga subir la ocupación, esto puede no suceder si la propensión marginal a consumir va en descenso.

La cita anterior puede representarse en tres desvíos del EK: (1) aumentos en la cantidad de dinero que no conducen a reducciones en la tasa de interés, (2) aumentos en la tasa de interés que no conducen a cambios positivos en la inversión, y (3) aumentos en la inversión que no conducen a mejoras en el empleo. Con respecto a (1), supongamos que la preferencia por la liquidez está aumentando más que la oferta monetaria. Si esto es así, entonces el exceso de dinero que posean las personas no se utilizará para comprar bonos, sino para atesoramiento; en otros términos, no es el motivo especulación el que prevalecerá, sino el motivo prevención. Como resultado, la tasa de interés no disminuirá. ${ }^{4}$

\footnotetext{
${ }^{4} \mathrm{El}$ ejemplo que generalmente se utiliza está asociado con la información que las personas tienen de la tasa de interés actual. Si ésta es muy baja, los agentes van a esperar que suba en un futuro muy cercano. Esto es equivalente a decir que esperarán que el precio de los bonos baje. Si tienen estas expectativas,
} 
En cuanto a (2), supongamos que el aumento en la oferta de dinero ha reducido satisfactoriamente la tasa de interés. Supongamos no obstante que los empresarios, sean por señales del mercado, por seguir al comportamiento de otros inversionistas, o por cualquier otra razón subjetiva, no tienen buenas expectativas de ventas futuras. Esto condicionará sensiblemente a la demanda de inversión. Por más que la tasa de interés haya caído -y que por consiguiente los créditos sea más baratos-, si los empresarios no tienen buenas expectativas de ventas futuras, la inversión no aumentará.

Supongamos finalmente que la inversión aumenta (3). Ello irá acompañado de un aumento en la ocupación primaria en las industrias de inversión $\left(\mathrm{N}_{2}\right)$. Ceteris paribus, y a través del multiplicador de la ocupación, también debería verificarse un aumento en la ocupación total. Empero, supongamos también que la propensión marginal a consumir disminuye por ejemplo, como efecto de una propaganda en tiempos de guerra a favor de restringir el consumo individual. En estos casos, un aumento en la ocupación en las industrias de inversión no incidirá favorablemente sobre la ocupación en las industrias que producen para el consumo (Keynes, 1936). En una situación como la descrita, las empresas que producen bienes de consumo percibirán, por un lado, la señal de que se ha producido un aumento en el empleo en las industrias de inversión, pero, por otro lado, percibirán una reducción inminente en la propensión marginal a consumir, lo cual afectará negativamente a sus expectativas de ventas futuras. Consecuentemente, no tendrán incentivos suficientes para contratar más trabajadores, haciendo así que la demanda total de empleo no aumente.

Complementariamente, en la medida en que se tengan en cuenta otros enfoques económicos se pueden ańadir nuevos escenarios al análisis. Por ejemplo, los monetaristas sostienen que si la cantidad de dinero sube más rápido que la tasa de crecimiento del ingreso nacional, entonces habrá un aumento en los precios. Los keynesianos también ven una posible consecuencia inflacionaria en esta política monetaria: si el aumento de la demanda agregada (causada por un aumento en la cantidad de dinero) termina excediendo el nivel de pleno empleo, entonces se producirá un aumento en los precios. La relación entre política monetaria expansiva e inflación también está predicha por la nueva economía clásica. Si los precios son completamente flexibles, si las expectativas del público son completamente racionales, y si las perturbaciones

luego no querrán salir a comprar bonos, dado ese exceso de dinero que poseen, sino que preferirán conservarlos. Se caerá en lo que Keynes denominó la "trampa de la liquidez". 
económicas reales son ruidos blancos, entonces el aumento de la oferta monetaria no producirá ningún aumento permanente en el empleo o en la producción. En el mejor de los casos, ese aumento será solo transitorio.

En general, el mecanismo que emerge luego de implementar una torta causal es un mecanismo cuyo comportamiento depende fuertemente de las acciones de las personas y de factores contextuales. El problema radica en que ninguno de ellos no son invariantes a lo largo del tiempo. Más precisamente, las acciones de las personas dependen de miríadas de factores. Un cambio en sus tomas de decisiones (el cual puede ser producto de un cambio en su formación de expectativas, en su manera de interpretar las señales del mundo, etc.) puede hacer que el mecanismo en cuestión ya no funcione. Esto es evidente en el EK. Las acciones de los empresarios dependen fuertemente de sus animal spirits. Los empresarios pueden no tener buenas expectativas de ventas futuras, y las razones pueden ser variadas: desconfianza en el presidente recién asumido, creencia de que la tasa impositiva se verá incrementada en los próximos periodos, creencia de que los aumentos en la demanda de sus bienes se debe a algo temporal y no a algo permanente, etc. No se puede obligar a las personas a actuar de ciertas maneras o a que formen determinadas expectativas. Lo que sí se puede hacer es crear instituciones para fomentar ciertas actividades y reducir otras, o crear seńales para mejorar las expectativas de los empresarios y de las familias. Pero hay una brecha entre estos intentos de manipulación y exigir determinadas conductas. Cuando la torta causal se arma, se espera que las personas actúen de una determinada manera y de que ciertos factores del contexto no cambien. Empero no hay seguridad de ello. Las personas no necesariamente van a actuar acorde a lo planeado. En una situación favorable, la política implementada puede funcionar satisfactoriamente debido a que las personas actúan acorde a lo establecido en la torta y a que las condiciones del contexto asumidas son las condiciones del contexto prevalecientes en el sistema objetivo. ¿Pero qué garantiza que éstas no cambien una vez que la política haya sido implementada? Las tortas causales propuestas por Cartwright y Hardie sirven para aquellos casos en los cuales no se espera que ocurran cambios estructurales o conductuales significativos. Por ejemplo, uno puede asumir con un buen grado de confianza que el rol que las madres juegan en un hogar típico de Tamil Nadu no cambiará una vez implementada la política. El problema estriba en qué factores uno asume que permanecerán invariantes. Suponer que las personas inmediatamente saldrán a comprar bonos una vez que reciban un incremento en sus saldos monetarios 
reales o que los empresarios siempre tengan buenas expectativas de ventas futuras es un camino directo al fracaso de la política. ${ }^{5}$

\section{LA IMPORTANCIA DE LA “ASIMETRÍA” EN LA TOMA DE DECISIONES}

Las tortas causales propuestas por Cartwright y Hardie proporcionan información respecto de lo que sucedería bajo circunstancias muy específicas. Sin embargo, estas tortas son incapaces de decirnos algo respecto de lo que sucedería si alguno de sus factores coadyuvantes no se cumpliesen, esto es, si la política se desviase de su curso esperado. En algunas situaciones estos desvíos pueden ser intrascendentes. Pero en otras situaciones pueden ser determinantes. $\mathrm{Y}$ esas desviaciones pueden ser suficientemente significativas como para marcar un punto de inflexión en la historia de la humanidad. Esto es lo que Taleb (2007) denomina "cisnes negros". Desde el punto de vista epistemológico, un cisne negro es una rareza, un evento que habita fuera del reino de las expectativas normales. Dado que es un suceso altamente improbable, es imposible de predecir. Asimismo, sus consecuencias son trascendentales, al punto de que cambian el rumbo de la historia. ${ }^{6}$ Ejemplos de ello son los éxitos de internet como Google, Facebook y YouTube, el atentado del 11-S, la crisis financiera de 2008 y, en general, casi todos los grandes inventos y descubrimientos de la historia humana.

De acuerdo con Taleb, en numerosas situaciones lo que no sabemos puede ser más importante que lo que sabemos. En la lógica del cisne negro prevalece la idea de prestar atención no a lo conocido, sino a lo improbable y a lo extremo, ya que son precisamente estos hechos los que terminan dominando nuestro mundo. Sin embargo, esto es justo lo que no se hace cuando se construyen las tortas causales. Dado que el objetivo central de esta estrategia es inferir un resultado con el mayor grado de certeza posible, el sistema bajo análisis recibe todo tipo de cierres y restricciones que garanticen el logro de ese resultado. El problema radica en que cualquier resultado alternativo o desviación es descartado, es decir, se convierte en una rareza. Peor aún, en algunas situaciones estas rarezas pueden tener consecuencias negativas e irreversibles.

\footnotetext{
${ }^{5}$ El hecho de que la implementación de una política pueda transformar ciertas condiciones de fondo y que como consecuencia de ello la política termine fracasando ha sido abordado, entre otros, por Lucas (1976) y Cartwright (2007).

${ }^{6}$ No toda rareza es un cisne negro. Para que un evento sea considerado como tal, el impacto que provoca en un determinado sistema debe ser importante.
} 
Conocer los desvíos de una torta es una buena base para la toma de decisiones de un hacedor de políticas, y en este sentido dicha toma de decisiones puede estar basada no sólo en las probabilidades de éxito de la política, sino también en sus consecuencias directas e indirectas. Esto es lo que Taleb (2012) denomina "asimetría". De acuerdo con Taleb, existen tres tipos de funciones subyacentes a relaciones causa-efecto: convexas (o asimetría positiva), cóncavas (o asimetría negativa) y lineal. Cuando hablamos de linealidad, decimos que el efecto es proporcional a su causa, es decir, que el cambio en la variable dependiente es proporcional al cambio en la variable independiente. Cuando hay asimetría, el cambio en la variable dependiente fluye en una proporción diferente de la independiente. Supongamos que estamos en el dominio de los positivos de un eje cartesiano. Dado un mismo punto de referencia, cuando una función es convexa lo que se pierde (valores ubicados a la izquierda de la función) siempre es menor a lo que se gana (valores ubicados a la derecha de la función). Cuanto más convexa sea esta función, mayor será la diferencia entre lo ganado y lo perdido. Lo inverso sucede con la función cóncava: lo que se pierde siempre es mayor a lo que se gana. Cuando la asimetría es positiva (o convexa), un agente tiene mucho para ganar y poco para perder. El resultado es claramente desconocido. Sin embargo, podemos hacernos una idea de las consecuencias de cada alternativa de decisión. La estrategia racional para Taleb no es la asignación de probabilidades a los eventos futuros, sino tomar decisiones basadas en las consecuencias: si el escenario involucra asimetría positiva, tendrá sentido seguir esa alternativa, ya que se tiene mucho para ganar y poco para perder. Si el escenario involucra asimetría negativa, lo racional será no actuar a favor de esa alternativa, ya que se tiene poco para ganar y mucho para perder.

Supongamos entonces que un país desea incrementar su PIB o renta nacional. Un camino posible es hacer uso del EK. Puesto que dicho mecanismo sólo funciona en circunstancias muy específicas, el hacedor de políticas debe cerciorarse de que se cumplan todas las condiciones y todos los factores coadyuvantes que conduzcan a incrementar la renta nacional ( $v . g r$, que los precios estén fijos, que la tasa de interés no sea demasiado baja, que los empresarios no tengan malas expectativas de ventas futuras, que existan recursos ociosos, etc.). Supongamos que estos requisitos se cumplen, y que el hacedor de políticas termina implementando la política. Supongamos asimismo que la política resulta ser exitosa: el aumento en la cantidad de dinero ha provocado un aumento en la renta nacional, sin que ello afecte al nivel de precios. 
Contento con este resultado, supongamos que el hacedor de políticas decide seguir incrementando a mansalva la cantidad de dinero, sin preocuparse por los efectos secundarios (o desvíos) que puede acarrear el uso sistemático de este mecanismo. Y uno de los efectos más nocivos de este mecanismo es la inflación. Así, supongamos que, de "manera inesperada", la enorme cantidad de billetes en la economía empieza a generar una presión en el nivel de precios. Una causa posible de esto puede ser el aumento en la demanda de activos financieros y de bienes y servicios que supera significativamente al aumento en la producción. Los empresarios aumentarán su nivel de producción, pero también sus precios. Esto va a ser el detonante para una espiral inflacionaria. Más precisamente, puesto que los precios de los bienes están aumentando, los sindicatos comenzarán a ejercer presiones para el alza de salarios. Esto aumentará el costo de los factores productivos, de modo tal que los empresarios terminarán aumentando nuevamente los precios. Si bien en un principio la inflación se correspondía con un aumento en el nivel de producción, es altamente probable que en algún momento esto se revierta. Asimismo, es posible que la espiral inflacionaria se mantenga aun en periodos recesivos o de caída de la renta nacional. La inflación de demanda que existía en un principio se transformó en una inflación de costos que desembocó en una inflación con recesión o "estanflación".

Así, una torta causal puede tener consecuencias positivas si todo sale de acuerdo con lo planeado. Sin embargo, el concepto de asimetría nos dice que más que apegarnos a esta única consecuencia, deberíamos también tener en cuenta qué desvíos o qué consecuencias alternativas pueden emerger en caso de que algo falle. Hay situaciones donde hay poco para ganar y mucho para perder. Y cuando esto suceda -esto es, cuando haya concavidad-, lo más prudente será no hacer uso de dicha política. A diferencia de Cartwright y Hardie -quienes eliminarían una torta causal en caso de que sus condiciones no sean implementables en un sistema objetivo-, el enfoque basado en la noción de asimetría sugiere que estas tortas se eliminen en caso de que sus consecuencias alternativas (aunque a veces improbables) sean sustancialmente dañinas para el sistema objetivo.

Alternativamente, existen casos en donde lo que se tiene para ganar es muy superior a lo que se tiene para perder, esto es, casos donde la asimetría es positiva. Cuando esto suceda, independientemente del éxito o fracaso de la política va a ser razonable su implementación, ya que no sólo se tiene control sobre lo que se pierde, sino que también las ganancias pueden ser 
ilimitadas. Tomemos como ejemplo aquellas políticas que consisten en combatir la desnutrición y malnutrición infantil. Lo que se "pierde" es algo conocido: son los recursos destinados en la implementación de la política. Lo que se gana es algo muy superior: una sociedad futura saludable, con menores índices de mortalidad infantil y con mayores oportunidades de llevar a cabo la vida que estas personas tienen razones para valorar. Las políticas que invierten en "buscar" cisnes negros son otro claro ejemplo de ello. ${ }^{7}$ Pensemos en las políticas que invierten en descubrir la cura contra enfermedades. Lo que se pierde es conocido y hasta controlable (ya que el hacedor de políticas puede decidir en algún momento cortar el flujo de recursos destinados a esta investigación). Pero lo que se gana con su descubrimiento es incalculable: así como es incalculable las vidas ganadas con el descubrimiento de la penicilina, también es incalculable las vidas que se ganarían el día que se descubra la cura contra el cáncer o contra el SIDA. Aquí existe otro fuerte contraste con el enfoque defendido en Cartwright (2012) y Cartwright y Hardie (2012). Para los autores, una política debe ser implementada sólo cuando se cumplan todas las condiciones estipuladas en las tortas lógicas. El enfoque de las políticas basadas en la asimetría de resultados pone mayor atención en las consecuencias que en la factibilidad del cumplimiento de estos factores coadyuvantes.

Así, si bien se reconoce el hecho de que conocer los factores coadyuvantes es de importancia clave para el éxito de una política, también lo es conocer las consecuencias alternativas de la misma. $\mathrm{Y}$ al conocerlas, la toma de decisiones puede cambiar sustancialmente. Conocer qué acervo de factores contribuyen al éxito de una política es solo una parte de la historia. Sin un conocimiento de las consecuencias alternativas el hacedor de políticas puede ir de una situación levemente complicada (v.gr., bajo crecimiento del PIB) a una situación crítica ( $v$.gr., estanflación). Asimismo, puede que no todos los factores coadyuvantes se cumplan, pero si la situación es de una asimetría positiva muy fuerte (como puede ser una política de urgencia donde el peor resultado ya está prácticamente asegurado), es razonable, bajo la propia lógica de la asimetría, que un hacedor de políticas decida igualmente implementar la política pertinente.

\footnotetext{
7 En este caso estaríamos hablando de cisnes negros positivos: aquellos que generan un beneficio incalculable. Estos se diferencian de los cisnes negros negativos, como las crisis económicas, las guerras, etc. Véase Taleb (2007).
} 


\section{CONSIDERACIONES FINALES}

En el enfoque de Cartwright y Hardie se considera que para que una política funcione aqui es necesario abstraer el principio causal de la manera correcta (investigación vertical), así como también éste debe ser complementado con un conjunto de factores coadyuvantes (investigación horizontal). Sin embargo, dicho enfoque ignora la posibilidad de que una política pueda fallar o desviarse de su curso previsto. En algunas situaciones estos desvíos pueden ser insignificantes. Pero no siempre es así. A veces las consecuencias pueden ser sustancialmente desproporcionadas respecto de la política pensada, y este resultado asimétrico puede ser tanto positivo como negativo.

Debido a esto, en el presente artículo se ha argumentado que el conocimiento de los resultados alternativos de una política resulta ser tan importante como el conocimiento de principios causales abstractos y de factores coadyuvantes que hacen que una política funcione aqui. Al tomar como punto de referencia el conocimiento de resultados alternativos, la toma de decisión estará sesgada por la asimetría de los resultados. Si un escenario involucra una asimetría positiva (es decir, si hay mucho para ganar y poco para perder), entonces sería coherente implementar la política. Del mismo modo, si un escenario involucra una asimetría negativa (es decir, si hay mucho para perder y poco para ganar), entonces, independientemente de las chances de éxito, lo coherente sería descartar dicha política. No se niega la importancia de realizar tanto la investigación vertical como la horizontal; de hecho el enfoque propuesto en el presente trabajo debería ser entendido como complementario a la tesis de Cartwright y Hardie. El problema estriba en que la sola información de factores causales abstractos y de factores coadyuvantes puede ser muchas veces insuficiente para una buena toma de decisiones.

IVAROLA, L. Alternative consequences: their importance in policy implementation. Trans/form/ação, Marília, v. 42, n. 2, p. 195-212, Abr./Jun., 2019.

ABSTRACT: The present paper examines the different kinds of knowledge required for correct policy implementation. Cartwright and Hardie (2012) have criticized the evidence-based policy approach for being grounded on inductive inferences. They propose an alternative approach where, on the one hand, the causal principle or policy variable is abstracted as much as possible in order to overcome external validity problems, and, on the other hand, this principle is complemented with a set of supporting 
factors which refer to very specific aspects of a target system. While this approach provides a good basis for formulating a policy, it omits a central issue: the alternative consequences that derive from a policy. More precisely, Cartwright and Hardie's approach aims to collect information in order to assemble a policy that works here. However, it says nothing about the cases where a policy deviates from its desired path. The problem arises when such deviations lead to transcendentally negative consequences. It will be argued that knowledge of a policy's alternative consequences are just as important as the knowledge of central and support factors. A policy-maker's decision will then depend not only on a policy's chances of success, but also on the asymmetry of its results (Taleb, 2012).

KEYWORDS: Policy implementation. Asymmetry. Alternative results. Evidence-based policy

\section{REFERENCIAS}

BANCO MUNDIAL. Tamil Nadu and child nutrition: a new assessment. Washington, DC: World Bank, 1995.

. Bangladesh integrated nutrition project: project performance assessment report. Report n. 32563. Washington DC: World Bank, 2005.

CARTWRIGHT, N. How the laws of physics lie. Oxford: Oxford University Press, 1983.

. Hunting causes and using them: approaches in philosophy and economics.

Cambridge: Cambridge University Press, 2007.

. Presidential address: will this policy work for you?: predicting effectiveness better: how philosophy helps. Philosophy of Science, v. 79, n. 5, p. 973-989, 2012.

CARTWRIGHT, N.; HARDIE, J. Evidence-based policy: a practical guide to doing It better. Oxford University Press, 2012.

KEYNES, J. La teoría general de la ocupación, el interés y el dinero. Buenos Aires: Fondo de Cultura Económica, 1936.

MACKIE, J. Causes and conditions. American Philosophical Quarterly, v. 2, n. 4, p. 245-264, 1965.

LUCAS, R. Econometric policy evaluation: a critique. Carnegie-Rochester Conference Series on Public Policy, v. 1, n. 1, p. 19-46, 1976.

TALEB, N. El cisne negro: el impacto de lo altamente improbable. Buenos Aires: Paidós, 2007.

. Antifrágil: las cosas que se benefician del desorden. Buenos Aires: Paidós, 2012.

Recebido: 04/03/2017

Aceito: 07/11/2017 
IVAROLA, L. 